\title{
SEQUENTIAL SAMPLING \\ TO MEASURE THE INFILTRATION RATE WITHIN RELATIVELY HOMOGENEOUS SOIL UNITS
}

\author{
A. Stein, J. Bouma, S.B. Kroonenberg \& S. Cobben, Wageningen
}

\section{Summary}

The statistical prediction techniques Trend Surface Analysis, Kriging and Cokriging are regularly used to provide predictive single-value soil maps. Kriging and Co-kriging perform well in situations where regionally distributed variables show clear spatial structure. If the amount of variation of the variables under study is relatively small, however, no gain in precision is gained by Kriging and Co-kriging, as compared with simply averaging over an area or by applying Trend Surface Analysis. This study was carried out on an older terrace of the Allier river in Central France where attention was focused on the infiltration rate (Inf). Kriging $\log (\mathrm{Inf})$ values did barely improve the predictions as compared with Trend Surface Analysis, whereas deleting part of the observations did not result in serious changes of predictions. A satisfactorial first approach was obtained by regarding the observations as being independent. To determine the sample size which provides sufficient information with respect to the soil characteristic under study use can be made of the sequential t-test as is illustrated.

ISSN 0341-8162

(c) 1989 by CATENA VERLAG,

D-3302 Cremlingen-Destedt, W. Germany

$0341-8162 / 89 / 5011851 /$ USS $2.00+0.25$
Application of this test resulted in eight measurements which represented a saving of $70 \%$ as compared with the original standard scheme.

\section{Introduction}

Introduction of environmental laws in various countries is associated with the need for quantitative expressions for environmental land characteristics. Whether or not certain normative levels are exceeded may be a crucial factor in court, where a quantitative statistical analysis is bound to have a higher impact than a comparative qualitative assessment. More emphasis on measurement has, however, major implications for soil characterization programs to be executed in the field by soil survey personnel. The associated costs may be prohibitively high and development of optimal sampling programs within particular land units is therefore important. Recent literature suggests the need for a preliminary sampling program to establish patterns of spatial variability to be followed by the sampling program itself in which the number of samples is optimized while one takes into account the obtained spatial variability structure and the required accuracy. Statistical prediction techniques, such as Kriging and Co-kriging can then be used to efficiently 
predict values for any location in the area (e.g. CORSTEN 1985).

However, even a preliminary sampling program can be rather time consuming when complex measurements are involved. This study is concerned with measurement of the infiltration rate, a land characteristic for which measurements are rather complex and time consuming. Rather than to proceed directly with the execution of a standard preliminary sampling program, which may require at least some thirty measurements, we have explored the possibility ot use geological and pedological descriptions of the land unit to be characterized, to possibly reduce the number of measurements. As it turns out, fortunately, some land units have soils or soil horizons with a low spatial variability. Much is gained if this can be established without an extensive preliminary sampling program involving the uncritical application of a standard geostatistical sampling procedure.

\section{Study area}

The study was carried out on one of a flight of nine fluvial terraces of the Allier river in the Limagne Graben in the French Central Massif. The sediments of the Allier river and its terraces comprise gravels, sands and clay, mainly of granitic-gneissic and basaltic provenance (KROONENBERG et al. 1982). The terraces are numbered according to French usage from $\mathrm{Fz}$ (youngest) to $\mathrm{Fs}$ (oldest). The bulk of the terraces consists of coarse gravel beds and sand lenses of several metres in thickness, but the uppermost 1 or 2 metres are usually clayey to sandy. Weathering of the volcanic components and progressive clay illuviation lead to a substantial increase in clay content from the soils of the lower, younger terraces to those of the higher, older ones (FEIJTEL et al. 1988). Variability of the soil infiltration rate from one terrace to another, therefore, is mainly dependent upon soil age. Data on spatial variability of infiltration rates within the youngest $\mathrm{Fz}$ terraces suggest a strong dependence upon soil texture, which is controlled in turn by sedimentation patterns. However, in the older Fv terrace segment, being studied here, little variability in soil texture could be detected in the field. As impeded drainage is one of the main limiting factors of the soils of this terrace, spatial variability of infiltration rate was investigated in this study, using geostatistical methods.

\section{Sampling and statistical methods}

The sampling design used here was similar to stratified sampling (STEIN et al. 1988). Different river terraces were distinguished as major land unit by means of geological and pedological descriptions. Within land units a fixed sampling design was followed, which was determined in advance.

Infiltration rates were measured with the standard double-ring infiltrometer (FAO 1979). Measurements were continued until a steady infiltration rate was reached for a period of at least one hour. A total of twenty-eight measurements were made in the top of the Bt-horizon at a depth of $30 \mathrm{~cm}$ below surface following a pre-determined grid (fig.1). This number was considered to be a compromise between the minimum number required to apply Kriging and Co-kriging and the maximum number that could reasonably be sampled in the available time. Four- 


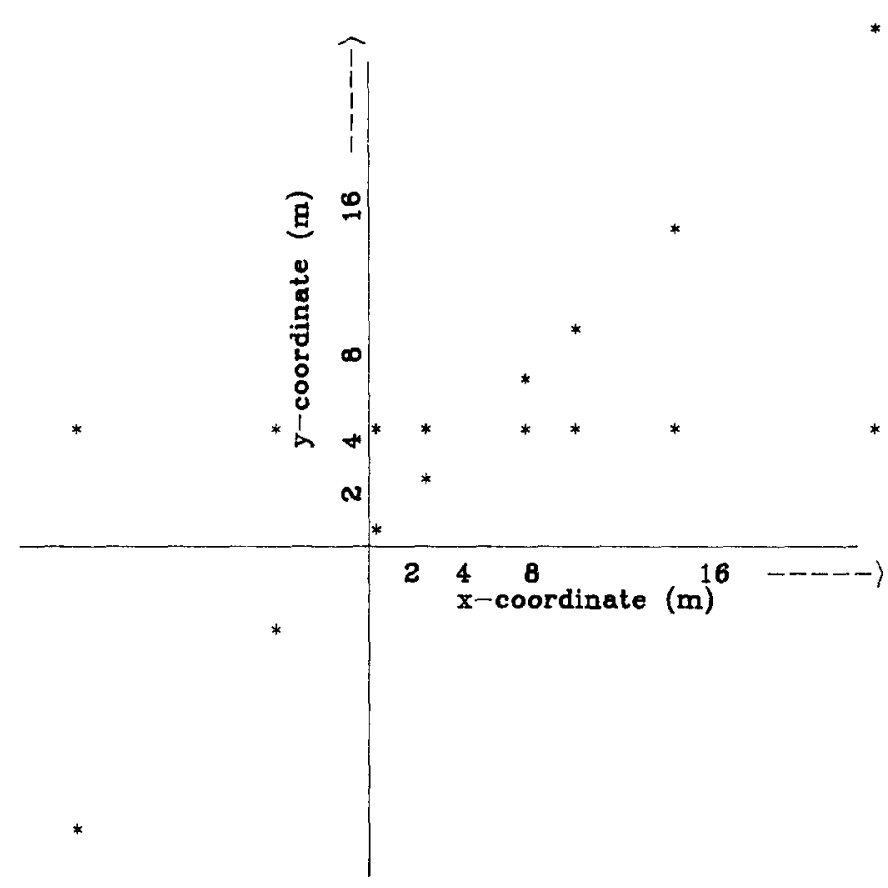

Fig. 1: Central part of the sampling scheme.

teen measurements were carried out in two directions on grid nodes with distances that increased exponentially from the centre $(2 \mathrm{~m}, 4 \mathrm{~m}, 8 \mathrm{~m}, \ldots, 128 \mathrm{~m})$. Fourteen additional measurements were carried out perpendicular to these directions at a distance of $2 \mathrm{~m}$ from a $2 \mathrm{~m}$ node, at a distance of $4 \mathrm{~m}$ from a $4 \mathrm{~m}$ node, etc. In all, therefore, a total of twentyeight measurements were obtained on two transects crossing with an angle of $45^{\circ}$.

Observations on infiltration rate (Inf) are reduced to their logarithmus $(\log (\operatorname{Inf}))$, to obtain a population which is approximately normal (fig.2). Cokriging was not considered, as the simple soil variables were only slightly correlated with infiltration rate (tab.1).

The statistical prediction techniques
Trend Surface Analysis (TSA) and Kriging are well described elsewhere (e.g. WATSON 1972; DELFINER 1976). Two different measures are used to evaluate the quality of predictions of infiltration rates for locations where no measurements were made:

1. The Mean of Squared Errors (MSE). Predictions $t_{i}(i=1, \ldots, n)$ are carried out in each observation point on the basis of the $\log (\operatorname{Inf})$ observations $y_{i}$ at all the remaining $(n-1)$ points. A measure of the quality of the prediction is given by:

$$
M S E=1 / n * \sum_{i=1}^{\mathrm{n}}\left(t_{i}-y_{i}\right)^{2}
$$

2. The Mean Variance of Prediction errors (MVP). As $t_{i}(i=1, \ldots, n)$ is 


\section{Frequency Histogram}

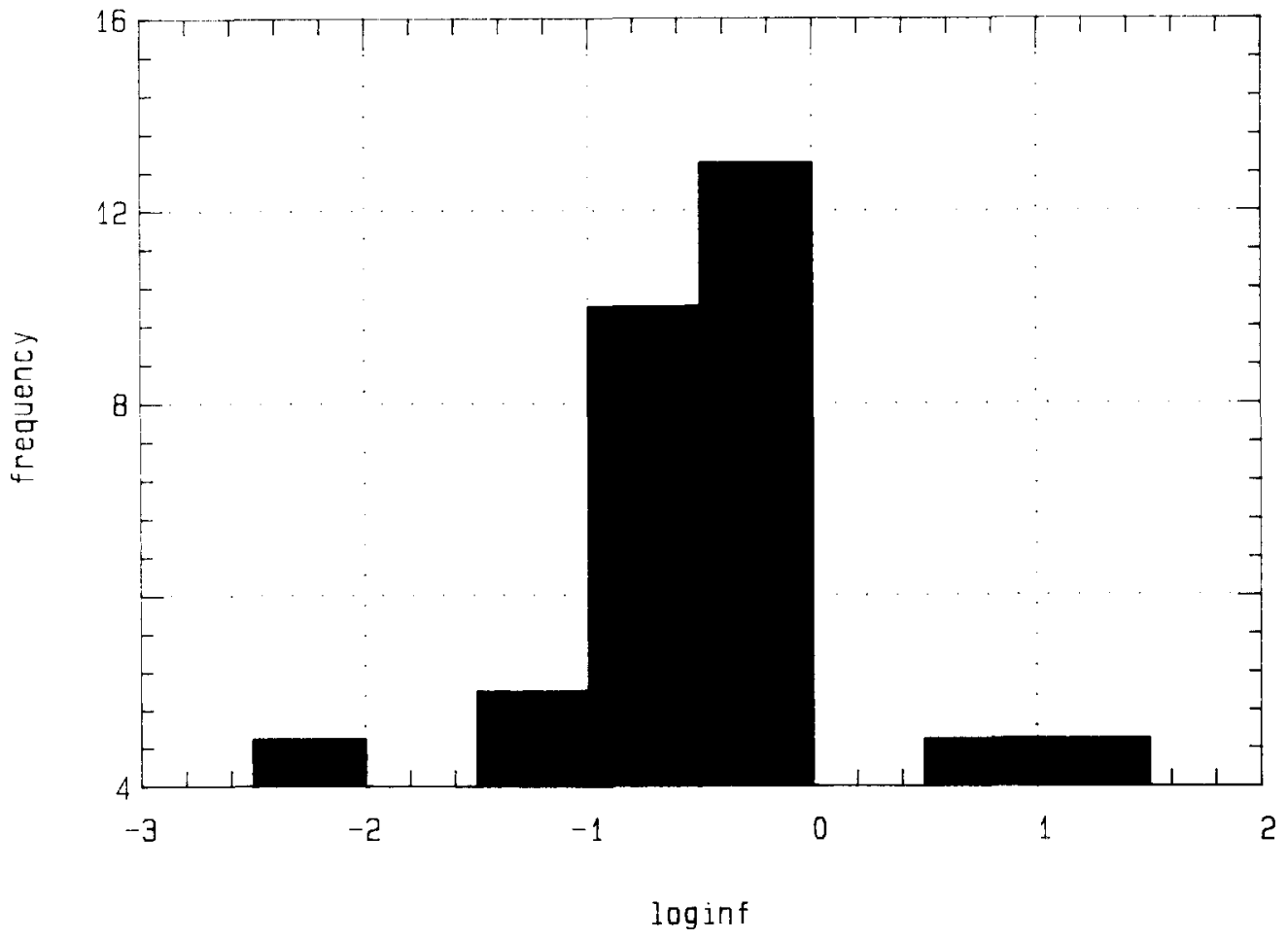

Fig. 2: Histogram of $\log (\operatorname{Inf})$ values.

\begin{tabular}{||lr|c|c|c||}
\hline & & & & \\
& & Mean & Standard Deviation & Correlation \\
\hline Log(Inf) & $\mathrm{cm} / \mathrm{day}$ & -.53 & .59 & 1.00 \\
Thickness Bt & $\mathrm{cm}$ & 46 & 9.55 & -.05 \\
Bulkdensity Bt & & 1.86 & .13 & -.21 \\
Relative height & $\mathrm{m}$ & 1.02 & .35 & .32 \\
Shearstress & & 71 & 20 & -.15 \\
Fraction Mottles & $\%$ & 16 & 11 & .12 \\
\hline
\end{tabular}

Tab. 1: Mean and Standard deviation of different variables in the study, as well as the correlation coefficient with $\log (\operatorname{Inf})$. 
the (stochastic) predictor in the $i^{\text {th }}$ observation point $\underline{y}_{0 i}(i=1, \ldots, n)$ of the $\log (\operatorname{Inf})$ value if it would have been measured, the MVP is defined as

$$
M V P=1 / n * \sum_{i=1}^{\mathrm{n}} \operatorname{var}\left(\underline{t}_{i}-\underline{y}_{0 i}\right)
$$

where $\operatorname{var}\left(\underline{t}_{i}-\underline{y}_{0 i}\right)$ is given by the formula for the prediction error variance under the prediction model being used, i.e. Kriging or TSA.

Two ways were chosen for investigating the differences in prediction performance by the two methods.

i) Values in the observation points were predicted disregarding the $\log (\operatorname{Inf})$ observations in those points and yielding the MVP. Afterwards differences between prediction and measurement were used to calculate the MSE.

ii) Predictions were carried out for 100 randomly located points within the sample area and the standard deviation of prediction error, being the square root of prediction error variance, was calculated.

Observation density was changed as follows. The complete set of 28 observations was reduced by four observations at a time, beginning from the centre, until the four outermost observations remained. A neighbourhood consisted of the observations in the reduced observation set, so its size changed proportional to the changing observation density.

A question we wanted to answer concerned the number of observations necessary to reach a predetermined precision.
This question was reformulated as: if after, say, m measurements some hypothesis is formulated concerning the distribution of the variable under study, is there any need to continue sampling, or can we stop. To investigate the need for additional observations in carrying out a pilot study, use was made of the sequential t-test (WALD 1948, KENDALL \& STUART 1973, see Appendix). In this test, a hypothesis concerning the distribution of the variable under study has to be formulated, which after some measurements is accepted or rejected. In this study we hypothesized the mean $\mu$ of the $\log (\operatorname{Inf})$ values to be equal to some value $\mu_{0}$. This hypothesis is rejected if $\left|\mu-\mu_{0}\right|>\delta_{1} * \sigma$. Here $\sigma$ is the standard deviation and $\sigma_{1}$ is some value to be determined in advance. The value of $\sigma_{1}$ depends on the allowed tolerance of the soil characteristic under study. In this case, the standard deviation is unknown in advance, so a slight modification of the sequential t-test was used. The method is considered to be an alternative to nested sampling (WEBSTER 1985).

When Kriging was applied, generalized increments were used to determine the degree of the trend and to obtain estimates for the coefficients of the generalized covariance function (STARKS \& FANG 1981). Three cases were considered: a $0^{\text {th }}$ order polynomial trend with generalized covariance function $g(h)=$ $\sigma_{0}+\sigma_{1} * h$, a $1^{\text {st }}$ order polynomial trend with generalized covariance function $g(h)=\alpha_{0}$ (a pure nugget effect) and a $1^{\text {st }}$ order polynomial trend with generalized covariance function $g(h)=\alpha_{1} * h$. Higher order trends were not taken into account because of the supposed homogeneity of the terrace segment, other models for the generalized covariance 


\begin{tabular}{|c|ccc|c|}
\hline Degree trend & \multicolumn{3}{|c|}{ Estimated coefficients } & Mean rank \\
& $\alpha-0$ & $\alpha-1$ & $x-3$ & \\
\hline 0 & 0.2778 & -1.244 & - & 40.54 \\
1 & 0.4136 & 0 & 0 & 41.75 \\
& 0 & -5.339 & 0 & 45.21 \\
\hline
\end{tabular}

\begin{tabular}{|c||cc|cc|}
\hline \multicolumn{1}{|c||}{} & \multicolumn{2}{c|}{ MSE } & \multicolumn{2}{c|}{ MVP } \\
nr. obs. & Kr & TSA & Kr & TSA \\
\hline 28 & .67 & .36 & .31 & .47 \\
24 & .72 & .31 & .33 & .47 \\
20 & .70 & .38 & .35 & .47 \\
16 & .77 & .47 & .39 & .48 \\
12 & .95 & .62 & .43 & .49 \\
8 & 1.16 & .93 & .52 & .51 \\
4 & .77 & .57 & .84 & .60 \\
\hline
\end{tabular}

\begin{tabular}{|c||cc||}
\hline \multicolumn{1}{|c||}{} & \multicolumn{2}{|c|}{100 points } \\
nr. obs. & Kr & TSA \\
\hline 28 & .40 & .47 \\
24 & .40 & .47 \\
20 & .40 & .47 \\
16 & .41 & .48 \\
12 & .41 & .49 \\
8 & .44 & .51 \\
4 & .56 & .56 \\
\hline
\end{tabular}

function did not lead to an admitted solution. The trends were compared by average ranks of the squared increments obtained with converged coefficients.

\section{Results and discussion}

The $\log (\operatorname{Inf})$ values are on the average -0.53 , with an individual standard deviation of 0.59 . This corresponds with an infiltration rate of about $4 \mathrm{~mm} / \mathrm{day}$, which is a very low value.

The lack of variability in infiltration rates in this terrace segment may be the result of strong clay illuviation, as well as of a decrease in the textural contrast between sandy and clayey parent materials due to profound weathering of the
Tab. 2: Estimated coefficients of the generalized covariance function for $a 0^{\text {th }}$ and a $1^{\text {st }}$ order trend and the mean rank of the squared increments.
Tab. 3: MSE and MVP values obtained by Kriging $(\mathrm{Kr})$ and Trend Surface Analysis (TSA), respectively, for the 28 observations used as a test set.

Tab. 4: MVP values obtained by Kriging ( $K r)$ and Trend Surface Analysis (TSA), respectively, for 100 randomly located points in the study area.

volcanic components in the sands.

For $\log (\ln f)$ the squared increments in the observation points were calculated. A $0^{\text {th }}$ order trend fitted slightly better than a $1^{\text {st }}$ order trend, as the mean rank increased from 40.54 to 41.75 , when the $0^{\text {th }}$ order trend is compared with a $1^{\text {st }}$ order trend and a pure nugget effect as a generalized covariance function (tab.2).

Using the overall mean of the $\log$ (Inf) observations as a prediction for the values in the 28 observation points yields a value for the MSE equalling 0.61. Predictions obained by Kriging are somewhat better, but differences are small (tab.3). The MSE values increase from .36 to .93 when the number of observations decreases from 28 to 8 . 


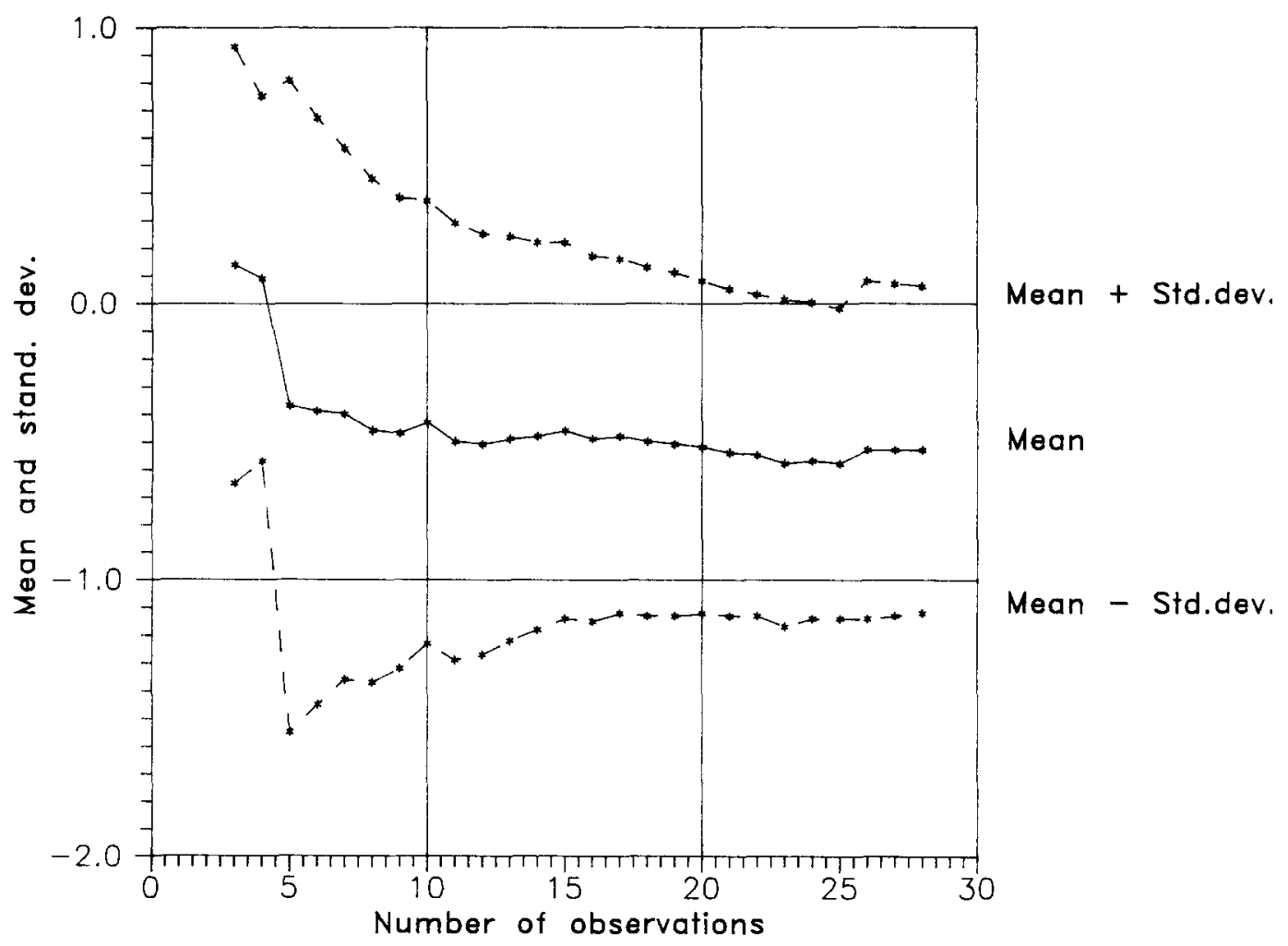

Fig. 3: Changes in $\log (\operatorname{Inf})$ mean and standard deviation as a function of the number of observations.

The change in the variance of the prediction error in the hundred randomly located spots is given in tab.4. No measurements were taken in these spots, so the MSE could not be calculated. The mean variance of the prediction error increases from .63 to 1.06 when the number of observations decreases from 28 to 8 .

As these changes in MSE and MVP values are considered small, the practical question must be raised now as to how an unnecessarily expensive exploratory survey of 28 measurements of infiltration rates can be avoided. Firstly, a geological and pedological analysis of the area to be studied is to be made, leading to a stratification of the area (here: the flight of terraces). For every stratum (terrace) a step by step increase in observation density can be made, where the observations are considered to be independent. Next, if a clear spatial structure in the soil variables can be distinguished and modelled, we can turn to the more advanced statistical prediction techniques as Kriging and Co-kriging.

Starting with the four outermost observations and the assumption that the observations were independent, the number and the density of observations was increased. It was noted that estimates for the mean and the standard devia- 


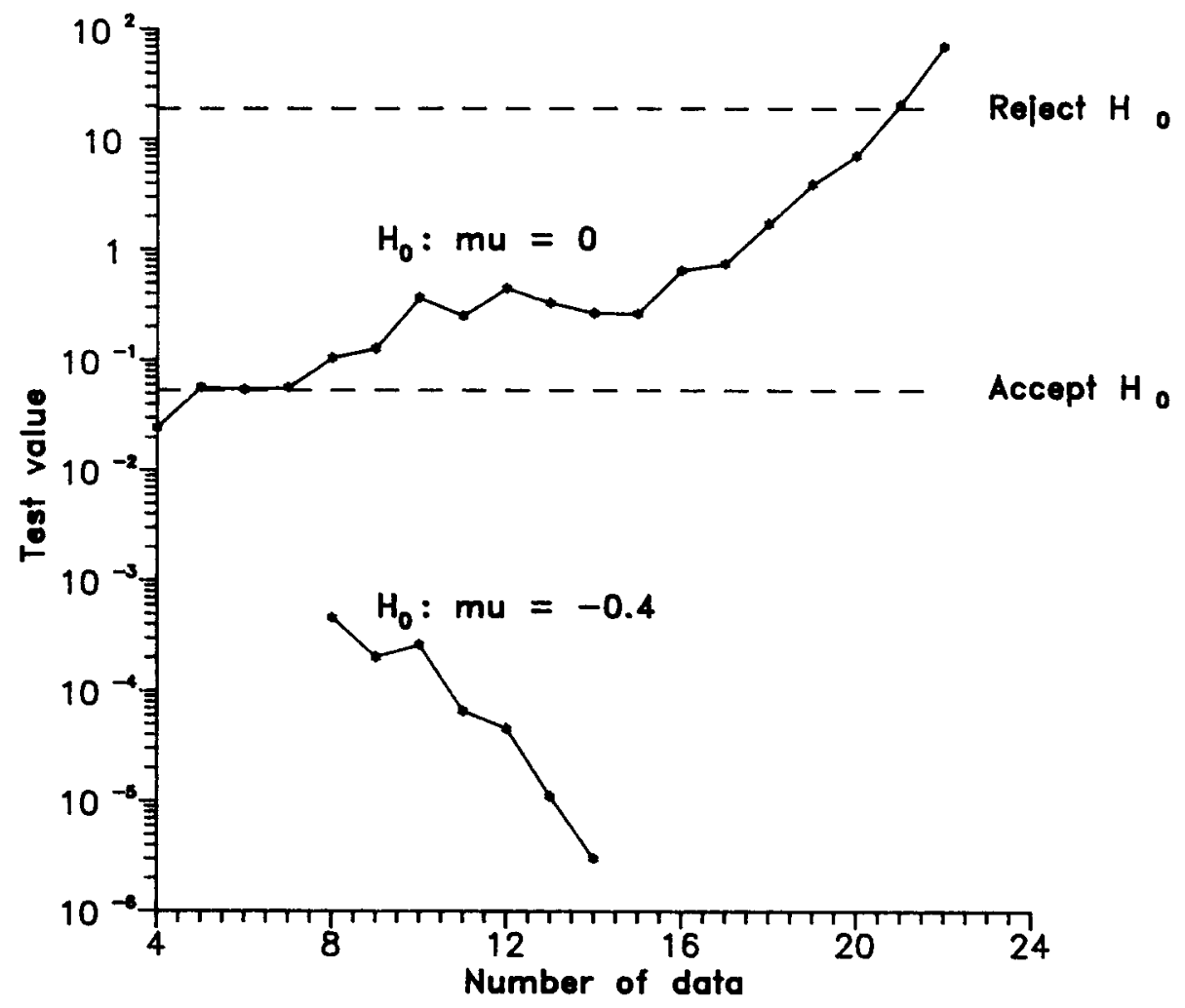

Fig. 4: Testing the hypotheses $H_{0}: \mu=0$ (after the fourth measurement) and $H_{0}: \mu=-0.4$ (after the eight measurement). Between the $H_{0}$-acceptance line and the $H_{0}$-rejecting line, applying to both hypotheses, is an intermediate zone.

tion only slightly change (fig.3). For the first four measurements we formulated the hypothesis $H_{0}$ that the mean value $\mu$ was equal to 0 , a hypothesis to be tested with error probability levels 0.05 for incorrectly deciding in favour of $\mathrm{H}_{0}$ as well as for incorrectly rejecting $H_{0}$ in favour of the value $\delta_{1}$ depending upon a feasible different value of the mean. In this case, a value of $\delta_{1}=1$ seemed to be appropriate, as a soil with an infiltration rate higher than $3 \mathrm{~cm}$ per day $(\log (\operatorname{Inf})$ $\approx 1$ ) was judged to be different from the hypothesized $1 \mathrm{~cm}$ per day $(\log (\operatorname{Inf}) \approx 0)$ from a practical point of view. Although at first the null hypothesis was likely to be accepted (fig.4), as soon as another measurement was taken the test value fell between the $H_{0}$-acceptance line and the $H_{0}$-rejecting line. In all 22 observations would be needed to finally reject $H_{0}$. After eight measurements were taken, $H_{0}$ was revised, leading to the hypthesis that $\mu$ be equal to -0.4 instead of 0 . This hypothesis was confirmed from the start, leading to the conclusion that with only 
eight observations enough information was obtained to decide in favour of $H_{0}$.

The procedure now can be generalized as follows. If, for different reasons, a low degree of variability is expected to occur within a stratum, observations are taken and sequentially followed, working from a low to a high observation density, by reducing the distances between observations step by step. Attention has to be focused on a hypothesis concerning the distribution of the soil characteristic under study. Locations for measurements are to be selected in advance, for instance randomly, or on predetermined transects or grids. The test value of the sequential test is calculated after each new measurement. As soon as the pre-defined limits based on confidence levels are reached, measurements are stopped, otherwise additional measurements have to be taken.

For our study, working from outside to the centre, eight measurements would have sufficed to yield estimates of infiltration rates that would not have been significantly different from results obtained by using the standard, exploratory survey of 28 measurements. The value of the standard deviation reduces from 1.18 to .60 . Both values are equivalent from a practical point of view, considering values for infiltration rate. The alternative procedure, discussed in this paper, which includes a geological and a pedological analysis before measurements are made, reduced costs by an estimated $70 \%$.

\section{References}

CORSTEN, L.C.A. (1985): Current statistical issues in agricultural research. Statistica Neerlandica 39(2), 156-168.

DELFINER, P. (1976): Linear estimation of non-stationary spatial phenomena. In: $M$. Guarascio et al. (eds.), Advanced geostatistics in the mining industry. 49-68. D. Reidel, Dordrecht.
FAO (1979): Soil survey investigations for irrigation. FAO Soils Bulletin 42, App. B-2, Rome.

FEIJTEL, T.C., JONGMANS, A.G., BREEMEN, N. VAN \& MIEDEMA, R. (1988): Genesis of two planosols in the Massif Central, France. Geoderma, 43, 249-269.

KENDALL, M.G. \& STUART, A. (1973): The advanced theory of statistics. Vol. 2 (3rd Edn.). Griffin, London.

KROONENBERG, S.B., MOURA, M.L. \& JONKER, A.T.J. (1988): Geochemistry of the sands of the Allier river terraces, France. Geologie en Mijnbouw 67, 75-89.

STEIN, A., HOOGERWERF, M. \& BOUMA, J. (1988): Use of soil map delineations to improve (Co-)kriging of point data on moisture deficits. Geoderma, 43, 163-177.

WALD, A. (1947): Sequential analysis. John Wiley, New York.

WATSON, G.S. (1971): Trend Surface Analysis. Jour. Mathematical Geology, v. 3, no. 3, 215226.

WEBSTER, R. (1985): Quantitative spatial analysis of soil in the field. Advances in soil science, $3,1-70$.

This paper is a contribution from the Dept. of Soil Science and Geology of the Agricultural University, P.O. Box 37, 6700 AA Wageningen, The Netherlands 


\section{Appendix}

\section{The sequential probability ratio test}

The Sequential Probability Ratio Test (SPRT) is designed to decide between two simple hypotheses. Suppose a random variable $z$ has a distribution $f(z, \mu)$, and we wish to test the hypothesis $H_{0}$ that $\mu=\mu_{0}$ against the alternative hypothesis $H_{a}$ that $\mu=\mu_{1}$. The test constructed decides in favour for either $\mu_{0}$ or $\mu_{1}$ on the basis of observations $z_{1}, z_{2}, \ldots$; we will suppose that if $H_{0}$ is true, we wish to decide for $H_{0}$ with probability with at least $(1-\alpha)$, while if $H_{a}$ is true, we wish to decide for $H a$ with probability at least $(1-\beta)$. We calculate the likelihood ratio $L_{m}$ as

$$
\begin{aligned}
L_{m} & =\frac{\text { Probability of observed values given } H_{a}}{\text { Probability of observed values given } H_{0}} \\
& =\prod_{i=1}^{m} \frac{f\left(z_{i}, \mu_{1}\right)}{f\left(z_{i}, \mu_{0}\right)}
\end{aligned}
$$

The procedure to follow is: continue sampling as long as $B<L_{m}<A$. Stop sampling and decide for $H_{0}$ as soon as $L_{m}<B$ and stop sampling and decide for $H_{a}$ as soon as $L_{m}>A$. It can be proven that $A \approx(1-\beta) / \alpha$ and $B \approx \beta /(1-\alpha)$.

As the standard deviation is unknown, we have to deal with a composite hypothesis, which is based upon weight functions. It is therefore reasonable to impose the following structure. For all $\mu$ satisfying $\left|\left(\mu-\mu_{0}\right) / \sigma\right|<\sigma_{0}$ it is preferred to accept the hypothesis $H_{0}$, and for all $\mu$ satisfying $\left|\left(\mu-\mu_{0}\right) / \sigma\right|>\sigma_{1}$ it is preferred to reject this hypothesis. We therefore specify three regions in the space $(\mu, \sigma)$, two in which $H_{0}$ is accepted and rejected, respectively,

Rejection if $\left|\mu-\mu_{0}\right| / \sigma>\delta_{1}$

Acceptance if $\mu=\mu_{0}$

while the remainder is an indifference region.

The likelihood ratio with appropriate weight functions can be formulated as

$$
\frac{1 / 2\left[\int_{0}^{\infty} \frac{1}{\sigma^{m}} \exp \left\{\frac{-1}{\sigma^{2}} \sum_{i=1}^{m}\left(z_{i}-\mu_{0}-\delta_{1} \sigma\right)^{2}\right\} d \sigma+\int_{0}^{\infty} \frac{1}{\sigma^{m}} \exp \left\{\frac{-1}{\sigma^{2}} \sum_{i=1}^{m}\left(z_{i}-\mu_{0}+\delta_{1} \sigma\right)^{2}\right\} d \sigma\right]}{\int_{0}^{\infty} \frac{1}{\sigma^{m}} \exp \left\{\frac{-1}{\sigma^{2}} \sum_{i=1}^{m}\left(z_{i}-\mu_{0}\right)^{2}\right\} d \sigma}
$$

where $m$ is the number of observations. The integrals appearing above were solved by means of a numerical integration routine.

$\begin{array}{ll}\text { Address of authors: } & \text { Dept. of Soil Science and Geology of the } \\ \text { A. Stein } & \text { Agricultural University } \\ \text { J. Bouma } & \text { P.O. Box } 37 \\ \text { S.B. Kroonenberg } & \text { 6700 AA Wageningen } \\ \text { S. Cobben } & \text { The Netherlands }\end{array}$

